\title{
Study of UK dental professionals reveals extent of occupational risk of SARS-CoV-2 infection
}

\section{A University of Birmingham-led study of over 1,000 dental} professionals has shown their increased occupational risk of SARSCoV-2 infection during the first wave of the pandemic in the UK. ${ }^{1}$

The observational cohort study, published on 3 June 2021 in the Journal of Dental Research, involved 1,507 Midland dental care practitioners. Blood samples were taken from the cohort at the start of the study in June 2020 to measure their levels of antibodies against SARS-CoV-2, the virus that causes COVID-19.

The team found $16.3 \%$ of study participants - which included dentists, dental nurses and dental hygienists - had SARS-CoV-2 antibodies, compared to just $6 \%$ of the general population at the time. Meanwhile, the percentage of dental practice receptionists, who have no direct patient contact, with SARS-CoV-2 antibodies was comparable to the general population, supporting the hypothesis that occupational risk arose from close exposure to patients.

The study also found ethnicity was a significant risk factor for infection, with $35 \%$ of black participants and $18.8 \%$ of Asian participants having SARS-CoV-2 antibodies, compared to $14.3 \%$ of white participants.

Blood samples were taken from participants three months later, in September 2020, when dental practices in England had re-opened with enhanced PPE and infection control measures in place, and once again in January 2021, six months after the start of the study, during the second wave of the pandemic when healthcare workers were being vaccinated.

The results showed that of those who had previous COVID-19 infection, over $70 \%$ continued to have SARS-CoV-2 antibodies both at three months and six months later, and they were at a $75 \%$ reduced risk of re-infection with the virus.

The study also demonstrated the immunological impact of COVID19 vaccination, with $97.7 \%$ of those without previous infection developing an antibody response at least 12 days after their first Pfizer vaccine. In those with evidence of previous infection, the antibody response was more rapid and higher in magnitude after a single dose of the Pfizer vaccine.

Furthermore, none of the cohort with a level of SARS-CoV-2 antibodies greater than $147.6 \mathrm{IU} / \mathrm{ml}$ in their blood tested positive for COVID-19 throughout the six-month period from the first to the final blood tests.

First author Dr Adrian Shields, of the University of Birmingham's Institute of Immunology and Immunotherapy, said: 'Understanding what an antibody test result means to an individual with respect to their risk of infection is essential to controlling the pandemic.

'Our study has taken the first steps in defining the level of antibody in a person's blood necessary to protect them from infection for six months. Furthermore, by comparing the antibody levels we have found in dentists to those contained in widely available reference material produced by the World Health Organisation, we hope the protective level we found can be easily confirmed and compared by other laboratories.'

Corresponding author Professor Thomas Dietrich, of the University of Birmingham's School of Dentistry, added: 'Critically, only 5.3\% of the cohort developed an antibody response that exceeded this threshold of $147.6 \mathrm{IU} / \mathrm{ml}$ following the first wave of the UK pandemic. This suggests that natural infection alone is unlikely to generate meaningful, durable herd immunity'.

Co-corresponding author Iain Chapple, Professor of Periodontology at the University of Birmingham and Consultant in Restorative Dentistry at Birmingham Community Healthcare Trust, said: 'Dental professionals are thought to be at high risk of exposure to SARS$\mathrm{CoV}-2$ because they routinely operate within patients' aerodigestive tract and regularly carry out aerosol-generating procedures that result in the production of airborne particles.

'Through our research, we have clearly shown that dental professionals were at increased occupational risk of exposure to SARS$\mathrm{CoV}-2$ prior to the new PHE guidance on PPE. The occupational health measures that have been put in place in general dental practice as a consequence of COVID-19 appear to remove that increased risk, however, this will need to be thoroughly investigated to see if they have successfully interrupted transmission of SARS-CoV-2 and other respiratory viruses.'

\section{Reference}

1. Shields A M, Faustini SE, Kristunas CA et al. COVID-19: Seroprevalence and vaccine responses in UK dental care professionals. J Dent Res 2021; doi: 10.1177/00220345211020270. 